\title{
Implementing Strategic Change in Manufacturing Organizations
}

\author{
John E. Oliver \\ Department of Management and Information Systems \\ Valdosta State College \\ Valdosta, Georgia \\ Gary B. Roberts \\ School of Business \\ Kennesaw College \\ Kennesaw, Georgia \\ and \\ Kerr Watson \\ Stokely Management Center \\ The University of Tennessee \\ Knoxville, Tennessee
}

One of the most common changes of strategy occurring in manufacturing organizations today is a new emphasis on product quality. When the mission of improved quality is operationalized, it involves the setting of quality improvement objectives and the design of programs to implement the new strategy. One of the most popular programs for implementing improved product quality is statistical process control (SPC) (2). SPC establishes current levels of quality variance in each stage of the manufacturing process in order to provide a basis for formulating improvement objectives. Then, continued measurements of quality variances are made to motivate and insure quality improvement through reduced variance at each stage in the process. Thus, SPC is used to formulate quality improvement objectives and to implement the quality improvement strategy, as well as to evaluate and control strategy implementation.

The implementation of a strategy of improved product quality using SPC provides a uniquely useful opportunity to study the complete strategy implementation process. The organizations in this study are all involved in the manufacture of components for the automobile industry and are all pursuing a strategy of improved product quality. They are implementing that strategy using statistical process control. The purpose of the study was to see whether managers in different types of organizations use different mechanisms to implement the new quality strategy.

\section{Strategy Implementation Mechanisms}

Galbraith and Nathanson (3), after an exhaustive literature review, listed nine mechanisms for implementing strategy - hierarchy, rules, goal setting, 
direct contact, interdepartmental liaison roles, temporary task forces, permanent teams, integrating roles, and integrating departments. To avoid duplication of terms and to clarify definitions, the following labels and definitions have been used in this research:

1. Chain of Command - The boss personally observes and directs the implementation of a new procedure through verbal instructions to the subordinates. When problems arise the boss usually directs behavior.

2. Rules and Standard Operating Procedures - Behaviors are specified in advance in the form of rules. Rules generally eliminate the need for further communication between the boss and subordinates unless specific problems arise. New procedures are implemented through the publication and distribution of standard operating procedures.

3. Targeting or Goal Setting - Behavior is not specified. Employees are allowed to select the way that they achieve the goals and objectives specified by the organization. New procedures are presented to employees in the form of targets and they are then free to choose the exact means to reach them.

4. Direct Contact - Individuals talk to each other when they are jointly affected by a problem. Problems are generally not referred up the chain of command but are resolved by the participants involved.

5. Liaison Roles - The liaison role is a typical example of a specialized role designed to facilitate communication between two interdependent departments and to bypass the long lines of communication involved in upward referral. For example, the engineering liaison in a manufacturing plant is part of the engineering organization but is physically located in the plant to serve the production organization. These roles link functional departments of the organization.

6. Temporary Task Force - The task force is a form of horizontal contact designed for problems across multiple departments. The task force is a temporary group made up of representatives from each of the affected departments. Some are full-time members; others may be part-time. It exists only as long as the problem remains. When a solution is reached participants return to their normal tasks.

7. Permanent Implementation Teams - Typically formed around frequently occurring problems. Teams are permanent groups which meet daily or weekly to discuss problems affecting the group. They solve all the problems which require commitments that they are capable of making. Larger problems are referred upward.

8. Integrating Roles - The response of the organization to the concern for decision quality is to create new roles in the organization structure. 
These roles are called integrating roles. The managers who occupy them do not supervise any of the actual work. Instead they assist those who do, so that the work is coordinated in the best interest of the organization. Some common titles for this role include: "materials manager," "product manager," "project manager," "program manager," and "unit manager".

9. Integrating Departments - Integrating departments consist of more than one person whose purpose is to assist those individuals and departments who do the actual work. Examples of such departments include "product management" and "project management" departments.

\section{Four Types of Organizations}

Four types of organizations were found empirically by Oliver $(7)$ in his initial studies with the Organization Description Questionnaire: hierarchic, professional, entrepreneurial, and group oriented. Each of these four types represents a different internal environment. As Campbell, Dunnette, Lawler, and Weich (1) suggested, the internal environments vary in terms of the amount of individual autonomy that is allowed to organization members, the degree of structure that is imposed, the reward orientation, the style of leadership exercised, and the way conflicts are managed.

In the hierarchic organization, individual autonomy is limited. Responsibility and authority are delegated in controlled doses by managers at higher levels of the hierarchy. Independence is controlled by job descriptions, rules, regulations, and guidelines. Initiative is limited by a system of standard operating procedures, specialization, and close supervision. The degree of structure is high with a great deal of formalization of procedures, centralization of decisions, and direct supervision exercised. Rewards are administered by superiors based on specific, well-defined, and objective criteria like time worked, items processed, errors, etc. The rewards are distributed on the bases of seniority, merit, and position. There is little consideration accorded subordinates in terms of warmth or support, and participation is generally kept to a minimum. Conflicts are seen as being bad for the organization and are discouraged. The most acceptable way of dealing with conflict is to appeal to higher levels in the hierarchy for resolution.

In the professional organization, a good deal of individual autonomy is granted. Responsibility is broad, with independence and initiative encouraged, so that the professional makes important decisions and takes action within the established body of knowledge and theory of the profession or technical specialty. Very little structure exists with the exception of formalization. Direct supervision is not needed since the code of behavior, professionalism, association, and peer review serve as substitutes for it. Decision-making is decentralized. Rewards are generally based on very general criteria and are often measured subjectively. The rewards generally go to those whose professional accomplishments are judged to be most significant. Leadership in the 
professional organization is characterized by a good deal of warmth, participation, and support, especially from colleagues. Conflict is encouraged in the professional organization because it leads to penetrating analysis and creative synthesis. Such conflict is seen as useful and good and is managed through a problem solving approach.

The entrepreneurial organization exhibits the greatest amount of individual autonomy with each individual given broad responsibility for accomplishment, complete independence and initiative, and the freedom to pursue goals by whatever means are appropriate. Structure is simple or non-existent with little or no formalization, centralization, standardization, or direct supervision. Rewards are based on the simple criterion of successful completion of the task or failure. Therefore, immediate feedback on success or failure is required and rewards follow automatically. Since the individual is left alone to complete the task, there is little support, warmth, or participation. Conflict is inherent in the task since the individual competes against himself, others, time, and other obstacles to achievement. Conflicts must be managed by the individual using whatever techniques are available. This usually means that solutions are forced by the individual, using drive or achievement motivation to overcome the obstacles. The word "entrepreneurial" is not used here to describe the simple structure of the typical small business but rather to describe the entrepreneur-like freedom or autonomy and the attendant risk that is widely distributed among organization members, as when jobs are enriched or commissioned sales people are given the freedom to set and pursue goals in their own way with only results being monitored.

The group organization replaces individual autonomy with group autonomy. While individuals may only have narrow responsibilities assigned, with independence and initiative limited, the group, functioning without supervision, takes on responsibility and initiative for the whole operation, which would be reserved for upper level management in the hierarchic organization. There is little or no direct supervision or centralization, but some structure is provided through a degree of formalized procedures. Rewards are generally based on specific, objectively measured criteria and distributed by some group incentive plan. Since leadership is emergent and democratic, a great deal of consideration is evident in the form of support, warmth, and ultimate participation. When conflicts occur, they may be perceived as either good or bad and are handled through group confrontation, discussion, and problem solving.

The four organization types are presented here as pure or ideal models based on Miner's (5) limited domain theories and Oliver's (7) empirical work. Obviously, the world of organizations is not so neat and simple. Most organizations contain some elements of all four types. For instance, hierarchy is a pervasive organization form and some elements of hierarchy will be found in almost all organizations. However, evidence indicates that most organizations can be classified into one of the four types (7). 
The four types are independent of industry. Firms within a given industry may possess any one of the four types of internal environments or even have different types in various units of the organization. An example of how different types may be found in one industry is the automobile industry. In the United States, most automobiles have traditionally been produced on the assembly line. Cars are assembled by workers who perform the same routine, highly specialized tasks over and over while under direct supervision. This is a predominately hierarchic organization.

In Zama, Japan, however, Nissans move down an automated assembly line where robots perform the routine tasks and workers are highly trained technicians and troubleshooters who work without close supervision. The workers' technical knowledge and professional orientation guide their behavior. This is a predominately professional organization.

In Kalmar, Sweden, Volvos are assembled by autonomous groups of workers in specially designed bays without immediate supervision. The self-directed groups plan, organize, and control production and are paid on a group incentive basis. This is a predominately group type internal environment.

Organization type, then, would seem to be a determinant of the kinds of strategy implementation mechanisms that an organization might use as well as a determinant of the effectiveness of a particular implementation method for a particular organization. The purpose of this study is to test whether managers in different types of organizations use different mechanisms to implement strategy. In Figure 1, the x's indicate which mechanisms are expected to be used in each organization type.

\section{The Study}

\section{Sample}

The data used in this study were gathered from a stratified random sample of 400 executives involved in a training program at a major southeastern university. The subjects represent 67 American and Canadian manufacturing organizations in the process of implementing a strategy of product quality improvement via a statistical process control program.

Usable responses were obtained from 181 of the subjects, yielding a $45.25 \%$ response rate.

\section{Measures}

Nine six-point Likert scale items similar to the one below were used to measure the usage of the nine strategy implementation mechanisms: 
Direct Contact - Individuals talk to each other when they are jointly affected by a problem. Problems are generally not referred up the chain of command but are resolved by participants involved.

$\begin{array}{lccccc}\begin{array}{l}\text { Never } \\ \text { Used }\end{array} & \begin{array}{l}\text { Rarely } \\ \text { Used }\end{array} & \begin{array}{l}\text { Somet Imes } \\ \text { Used }\end{array} & \begin{array}{l}\text { Often } \\ \text { Used }\end{array} & \begin{array}{l}\text { Very } \\ \text { Often } \\ \text { Used }\end{array} & \begin{array}{l}\text { Always } \\ \text { Used }\end{array} \\ 0 & 1 & 2 & 3 & 4 & 5\end{array}$

The Oliver Organization Description Questionnaire (OODQ) (7) was used to classify the respondents' organizations as hierarchic, professional, entrepreneurial, group, or some combination of the four. The OODQ is a forced-choice questionnaire which yields a hierarchic score, a professional score, a task score, and a group score. A total of fifteen items are scored for each domain scale so that possible scores range from 0 to plus 15 . The following is an example of an OODQ item:

1. In my work, duties are determined by

(a) management

(b) my professton or ocupation

(c) my work group

(d) me, based on the goal I am trying to accomplish

In addition to the two questionnaires, respondents reported their organization level as top level executive, middle manager, first line supervisor, or professional (engineer, consultant, etc.). Organization size was coded for each firm based on the number of employees, as listed in Standard and Poors Directory.

\section{Procedure}

Organizations were classified by highest OODQ score. Multivariate analysis of variance (MANOVA) and univariate ANOVA tests were conducted to determine whether there were significant differences in the reported usage of strategy implementation mechanisms in the four types of organizations, in different size organizations, and by organization level. Tukey's c.v. tests were used to determine which differences were significant. 


\begin{tabular}{|c|c|c|c|c|c|}
\hline \multirow[b]{2}{*}{ Mechanisms } & \multicolumn{4}{|c|}{$\begin{array}{l}\text { Mean Ratings of Usage of Strategy Implementation Mechanisms } \\
\text { In Four Types of Organizations }\end{array}$} & \multirow{2}{*}{$\begin{array}{l}\text { Total } \\
\text { Sample } \\
(n=181)\end{array}$} \\
\hline & $\begin{array}{l}\text { Hierarchy } \\
(n=117)\end{array}$ & $\begin{array}{l}\text { Professional } \\
(n=20)\end{array}$ & $\begin{array}{l}\text { Entrepreneurial } \\
\qquad(\mathbf{n}=\mathbf{3 0})\end{array}$ & $\begin{array}{l}\text { Group } \\
(n=14)\end{array}$ & \\
\hline Chain Of Command & 2.58 & 2.83 & 2.28 & 2.14 & 2.38 \\
\hline S.O.P.'s*** & $2.58 \mathrm{EG}$ & $2.83 \mathrm{G}$ & 1.94 & 1.43 & 2.42 \\
\hline Goal-Setting $* * *$ & 2.25 & 2.87 & $3.22 \mathrm{H}$ & $3.21 \mathrm{H}$ & 2.56 \\
\hline Direct Contact $* * *$ & 2.79 & $3.48 \mathrm{H}$ & $3.33 \mathrm{H}$ & $3.64 \mathrm{H}$ & 3.02 \\
\hline Interdep. Liaison & 2.42 & 3.00 & 2.28 & 2.86 & 2.49 \\
\hline Temp. Task Force* & 2.60 & 3.17 & 2.86 & 3.36 & 2.76 \\
\hline Perm. Imp. Team** & 2.29 & 3.09 & 2.61 & $3.36 \mathrm{H}$ & 2.51 \\
\hline Integrating Role & 2.34 & 2.22 & 2.44 & 2.79 & 2.32 \\
\hline Integrating Dept. & 2.03 & 2.04 & 2.08 & 2.14 & 2.05 \\
\hline
\end{tabular}

* Mean differences significant $p<.05$

** Mean differences significant $p<.01$

***Mean differences significant $p<.001$

$H=$ Significantly greater than Hierarchic sample $(p<.01)$

$E=$ Significantly greater than Entrepreneurial sample $(p<.01)$

$G=$ Significantly greater than Group sample $(p<.01)$

\section{Results}

Table 1 contains the mean scores of usage ratings for the mechanisms in each organization type, as well as the entire sample. Significant differences are summarized in the discussion section of this paper.

Table 2 lists the mean scores of usage ratings for the mechanisms in four different sized organizations. The differences are summarized in the discussion.

Differences in usage of the mechanisms by executives at each level in the organizations are reported in Table 3 and summarized in the discussion.

Above-average usage is shown in Figure 1 by circles (0). Circles which contain $x$ 's indicate that above-average use was hypothesized and confirmed. Seventeen of the twenty hypothesized above-average usages were confirmed. Three of those hypothesized were not supported by the data, and five unexpected above-average usages were reported.

\section{Discussion}

As Mintzberg (6) and others have documented, direct contact is the most popular mechanism for implementing strategy-ranking first in all four types of organizations, all sizes of organizations, and all levels. Apparently, managers and professionals still prefer face-to-face contact to get things done. Oddly enough, it is more often used in larger rather than smaller organizations; and first line supervisors prefer it.

The second most popular mechanism is the temporary task force. While this research does not clearly indicate why the task force is so popular, it can be said that it is the least disruptive, costly, and most flexible of the mechanisms. It was the second most-used mechanism in three of the four 
Table 2

\begin{tabular}{|c|c|c|c|c|}
\hline \multicolumn{5}{|c|}{$\begin{array}{c}\text { Mean Ratings of Usage of Strategy Implementation Mechanisms } \\
\text { in Four Sizes of Organizations' }\end{array}$} \\
\hline Mechanisms & $\begin{array}{l}\text { Very Large } \\
(n=65)\end{array}$ & $\begin{array}{l}\text { Large } \\
(n=64)\end{array}$ & $\begin{array}{c}\text { Medium } \\
(\mathrm{n}=34)\end{array}$ & $\begin{array}{c}\text { Small } \\
(n=18)\end{array}$ \\
\hline Chain of Command & 2.42 & 2.32 & 2.72 & 2.48 \\
\hline S.O.P.'s* & 2.18 & 2.14 & 2.07 & 2.18 \\
\hline Goal-Setting** & 2.83 & 3.00 & 2.93 & 2.40 \\
\hline Direct Contact** & 3.47 & 3.31 & 3.29 & 3.02 \\
\hline Interdep. Liaison & 2.89 & 2.74 & 2.71 & 2.10 \\
\hline Temp. Task Force* & 3.34 & 2.66 & 3.05 & 2.74 \\
\hline Perm. Imp. Team** & 3.16 & 2.55 & 2.91 & 2.32 \\
\hline Integrating Role & 2.44 & 2.52 & 2.04 & 2.41 \\
\hline Integrating Dept. & 2.39 & 2.08 & 1.99 & 1.57 \\
\hline
\end{tabular}

* Mean differences significant at $p<.01$

${ }^{* *}$ Mean differences significant at $p<.001$

IVery Large $=>50,000$ employees

Large $=10,000-50,000$ employees

Medium $=1,000-10,000$ employees

Small $=\langle 1,000$ employees

Table 3

\begin{tabular}{|lcccc|}
\hline \multicolumn{5}{c}{$\begin{array}{c}\text { Mean Ratings of Usage of Strategy Implementation Mechanisms } \\
\text { Reported by Four Levels of Respondents }\end{array}$} \\
$\begin{array}{l}\text { Top-Level } \\
\text { Mechanisms }\end{array}$ & $\begin{array}{c}\text { Mid-Level } \\
(\mathbf{n}=\mathbf{1 6})\end{array}$ & $\begin{array}{c}\text { Professional } \\
(\mathbf{n}=\mathbf{4 1})\end{array}$ & $\begin{array}{c}\text { First-Line } \\
(\mathbf{n}=56)\end{array}$ \\
Chain of Command & 2.64 & 2.45 & 2.39 & 2.45 \\
S.O.P.'s** & 1.58 & 2.32 & 2.27 & 1.93 \\
Goal-Setting** & 3.08 & 2.84 & 2.91 & 2.75 \\
Direct Contact** & 3.26 & 3.28 & 3.33 & 3.42 \\
Interdep. Liaison & 2.61 & 2.85 & 2.59 & 2.56 \\
Temp. Task Force & 2.93 & 3.00 & 3.17 & 2.66 \\
Perm. Impl. Teams* & 3.05 & 2.79 & 3.00 & 2.42 \\
Integrating Roles & 2.52 & 2.41 & 2.20 & 2.46 \\
Integrating Depts. & 2.19 & 2.04 & 2.21 & 2.03 \\
\hline
\end{tabular}

* Mean difference significant at $p<.01$

** Mean differences significant at $p<.001$

'Top-level $=$ Top level executives

Mid-level $=$ Middle managers

Professional $=$ Professional engineers, consultants, etc.

First-Line $=$ First line supervisors 


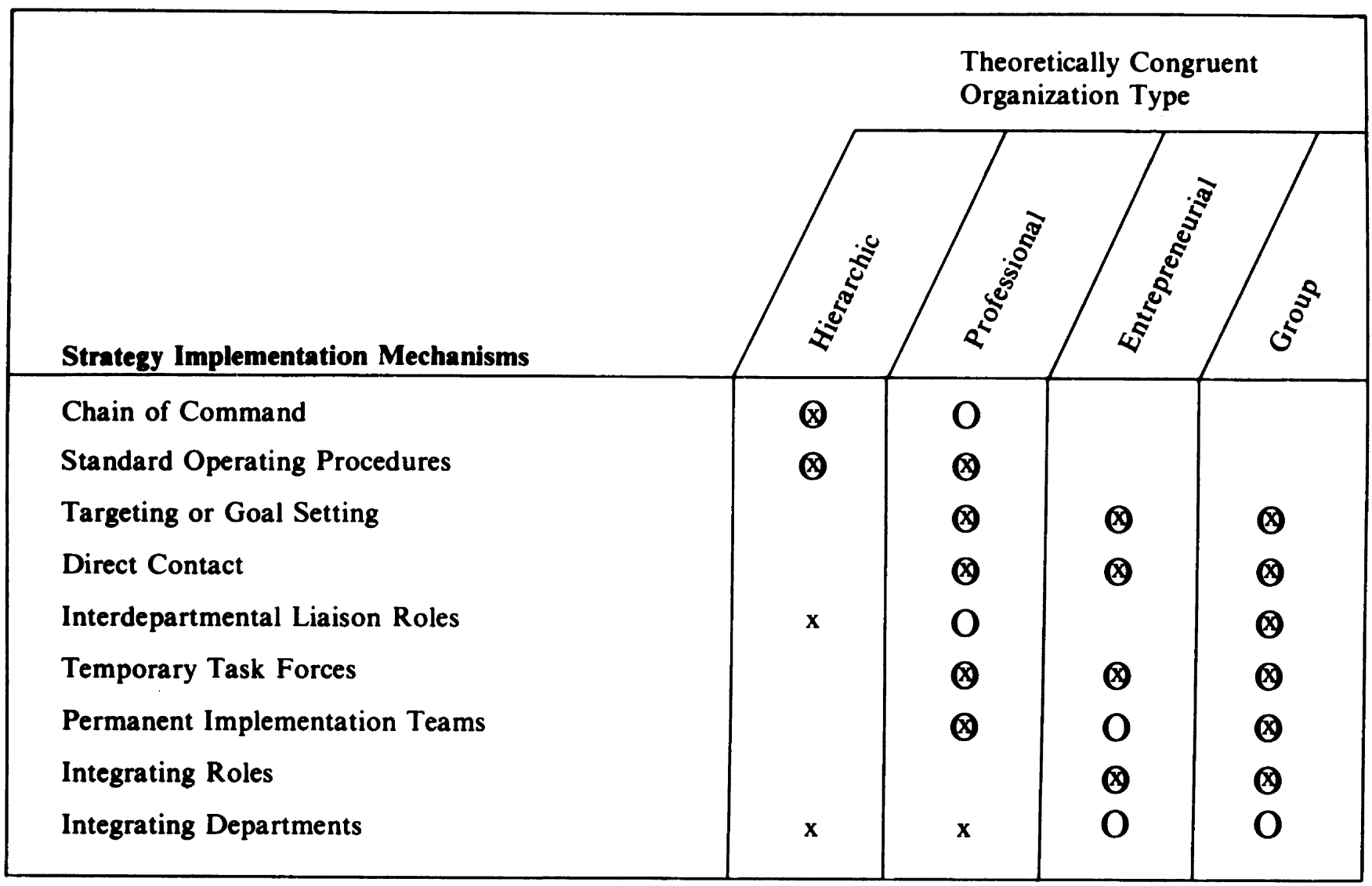

$\mathrm{x}=$ hypothesized above average usage

$\mathrm{O}=$ actual reported usage above the mean for the total sample

Figure 1

Hypothesized and Actual Reported Use of Strategy Implementation Mechanisms by Organization Type 
types of organizations and third most-used in the entrepreneurial type, while being most often used by professionals and mid-level managers.

Although goal setting was the third most-used mechanism overall, its use varied a great deal among the four types of organizations. It was second only to direct contact in entrepreneurial organizations where it was hypothesized to be most appropriate, but was eighth out of nine in hierarchic organizations and fifth in the professional type. Its unpopularity in hierarchies may be due to the difficulty of changing goals or redirecting emphasis away from their rigid and traditional, bureaucratic goals, policies, and procedures.

As expected, permanent implementation teams, which were fourth in popularity overall, were most used in the group type organizations where their use was rated equally with temporary task forces. The use of permanent teams was ranked third and fourth in professional and entrepreneurial organizations, respectively, but seventh in hierarchies. This low usage in hierarchies may reflect the reluctance of hierarchic managers to create long-lived committees which might become opposing forces or obstacles, or it may indicate that the established hierarchic structure is a sufficient and existing "permanent implementation team."

Interdepartmental liaison roles were ranked fifth out of nine in overall usage putting them at the midpoint of popularity among strategy implementation mechanisms in this study. Their use did not vary significantly by size or level of organization.

While standard operating procedures, which ranked sixth overall, were reportedly used most often in professional organizations, their use ranked third (tied with chain-of-command) in hierarchies. As expected, their use is most theoretically congruent with hierarchies and professional organizations and is supported by the data.

The reported use of chain-of-command, which ranked seventh out of nine overall, was also greatest for hierarchies as was hypothesized. Though this hypothesis was not supported statistically, the rank of third most popular does indicate some support for the notion that chain-of-command as a strategy implementation mechanism is primarily appropriate to the hierarchic organization. Perhaps the lack of clarity in this data is due to the pervasiveness of chain-of-command in all or most organizations.

The use of integrating roles and departments as strategy implementation mechanisms, at least in this sample, appears to be rare, with no significant differences among types, sizes, or levels of organizations.

This study indicated that as firms move through their life cycle, they may utilize different mechanisms. This study indicates that there may be a life cycle effect in the use of both temporary task forces and permanent implementation teams with small and large organizations using them less than the medium and very large. This could indicate that firms growing from small to medium size experience a reduction in the effectiveness of standard operating procedures and begin to "grope in groups." The teams work for awhile, but their effectiveness fades with time and growth. Then, as organizations grow 
from large to very large, they again turn to "group groping." The pattern of usage for SOP's is somewhat different, with significantly greater usage in small and very large firms than in medium and large ones. The use of goalsetting is just the opposite. Neither small nor very large firms use goal-setting to the degree that a medium or large one does. SOP's and goal-setting may be incongruent mechanisms which hinder each other or cannot be used effectively together.

\section{Conclusions}

The findings in this study have limited generalizability due to: the small number of professional, entrepreneurial, and group type organizations represented in the sample; the nature of the single strategy being implemented; and the fact that all of the firms are manufacturers in related industries. Further study is needed on a broader variety of organizations implementing a variety of different strategies in order to fill in the missing pieces of the puzzle. However, this study provides support for the general hypothesis that different strategy implementation mechanisms are used in different organizational types and sizes, as well as by executives at different levels of the organization.

\section{References}

1. Campbell, John P., Dunnette, Marvin D., Lawler, Edward E. III, and Weick, Karl E., Managerial Behavior, Performance, and Effectiveness (New York: McGraw-Hill, 1970), p. 393.

2. Deming, W. E. (1982) Quality Productivity, and Competitive Position. Cambridge: Massachusetts Institute of Technology, Center for Advanced Engineering Study.

3. Galbraith, J. and Nathanson, D. 1978 Strategy Implementation: The Role of Structure and Process, St. Paul, MN: West Publishing Company.

4. Lawrence, Paul R. and Jay W. Lorsch. Organization and Environment: Managing Differentiation and Integration. Boston: Graduate School of Business, Harvard University, 1967.

5. Miner, J. B. 1979. "Limited Domain Theories of Organizational Energy." C. C. Pinder and L.B. Moore (eds). Middle Range Theory and the Study of Organizations. Leiden, Netherlands: Martinus Nijoff: 273286.

6. Mintzberg, H. The Nature of Managerial Work. New York: Harper and Row, 1973.

7. Oliver, J. E., 1982. "An Instrument for Classifying Organizations." Academy of Management Journal. 25 (4): 855-866. 